\title{
Statistical LOS/NLOS Channel Model for Simulations of Next Generation 3GPP Networks
}

\author{
Rimvydas Aleksiejunas, Albert Cesiul, Kestutis Svirskas \\ Institute of Applied Electrodynamics and Telecommunications, Vilnius University, \\ Sauletekio Al. 9, Vilnius LT-10222, Lithuania \\ albert.cesiul@ff.vu.lt
}

\begin{abstract}
Understanding of line-of-sight and non-line-ofsight (LOS/NLOS) visibility conditions is important for radio wave propagation modeling when selecting empirical path loss model. For Monte Carlo statistical simulations a random generator of visibility states can be designed according to a given spatial probability density. In the current paper, a statistical LOS state model is derived based on ITU-R and 3GPP 3D LOS probability models suitable for 5G channel simulations. The generic exponential LOS probability model is improved by dividing the area into LOS and NLOS zones depending on the building density. The accuracy of LOS probability model has been tested against visibility predictions obtained from the digital building data over Manhattan city area.
\end{abstract}

Index Terms-LOS; NLOS; Wave propagation; Non line of sight; 3GPP; 5G.

\section{INTRODUCTION}

Line-of-sight or non-line-of-sight (LOS/NLOS) visibility conditions are required for radio wave propagation modeling to properly select empirical path loss model. For Monte Carlo statistical simulations a random generator of visibility states should be design according to a given spatial probability density. The methods of LOS state simulation are essential part in system level simulators such as SEAMCAT software tool [1] used by spectrum regulators. Statistical LOS probability function over distance can be derived from measurements and simulations using digital building height data. Various LOS probability approximations have been used to date, including ITU [2] and 3GPP [3] models. Such models apply the same LOS probability dependence on distance for a whole area of analysis. However, real urban territories represent city blocks with different building density and heights. Thus taking into account inhomogeneity of propagation environment would increase accuracy of LOS probability approximations. In the paper, a dual environment LOS probability model is proposed which approximates probability by splitting all area into two environments depending on the building heights: one which allows LOS propagation with higher probability and another environment - represented by lower LOS probability. Here we are concerned only with separation of propagation environments into LOS and NLOS conditions, avoiding further insights into propagation losses such as building

Manuscript received 18 March, 2018; accepted 30 July, 2018. penetration or diffraction.

The paper is organized in the following order: the next section reviews existing methods and algorithms for approximating LOS probability function in a spatially consistent manner. Section III introduces dual environment model and applies model approximation to urban area with Manhattan city building data. Finally, conclusions are drawn.

\section{LOS/NLOS State SimUlation MethodS}

\section{A. LOS Probability}

LOS probability determination procedures proposed by ITU [2] and 3GPP [3] 3D channel models are widely accepted in $4 \mathrm{G}$ and $5 \mathrm{G}$ network simulation studies. There exist several different LOS visibility probability models depending on the propagation scenario. For indoor mobile users, visibility conditions are determined based on the floor height [4]. For lower 4 floors or up to $12 \mathrm{~m}$, type 1 visibility probability is assumed according to ITU-R M.2135-1 report [2].

3GPP and ITU proposed LOS probability models can be approximated by the following distance dependence law

$$
p_{\mathrm{LOS}}(d)=\min \left(\frac{d_{1}}{d}, 1\right)\left(1-\mathrm{e}^{-\frac{d}{d_{2}}}\right)+\mathrm{e}^{-\frac{d}{d_{2}}},
$$

for urban scenarios and

$$
p_{\text {LOS }}(d)=d_{3} \mathrm{e}^{-\frac{d}{d_{4}}},
$$

for suburban areas, where coefficients $d_{\mathrm{i}}, i=\{1,2,3,4\}$ are empirical data fit parameters and $d$ is the distance in meters.

A modification of this $p_{\mathrm{LOS}}(d)$ model has been proposed in [5] with squared distance approximation verified for millimeter wave outdoor propagation in $28 \mathrm{GHz}$ band

$$
p_{\mathrm{LOS}}(d)=\left[\min \left(\frac{d_{1}}{d}, 1\right)\left(1-\mathrm{e}^{-\frac{d}{d_{2}}}\right)+\mathrm{e}^{-\frac{d}{d_{2}}}\right]^{2}
$$

\section{B. Spatial Consistency}

The importance of spatially consistent radio channel 
models including LOS/NLOS states in propagation modeling has been stressed in recent research funding programs for $5 \mathrm{G}$ wireless communications such as METIS and IRACON. Random distribution of LOS/NLOS states with a given distribution probability can be generated using autocorrelation function method in a similar way as used in shadow fading modeling [6]. As an alternative to autocorrelation method for LOS/NLOS visibility state generation is the interpolation of randomly generated visibility values over decorrelation distance $d_{\text {corr }}$ [7]. This algorithm consists of the following steps:

1. Generating grid of decorrelated complex random normal i.i.d. variables $Z_{m n}, m=[1, \ldots, M], n=[1, \ldots, N]$ over whole rectangular area as $M \times N$ matrix,

2. The complex normal random variable distribution at a given position $x, y$ can be interpolated from four nearest $Z_{m n}$ matrix points as

$$
\begin{gathered}
Z(x, y)=\sqrt{1-\frac{y}{d_{\text {corr }}}}\left(\sqrt{1-\frac{x}{d_{\text {corr }}}} Z_{00}+\sqrt{\frac{x}{d_{\text {corr }}}} Z_{10}\right)+ \\
+\sqrt{\frac{y}{d_{\text {corr }}}}\left(\sqrt{1-\frac{x}{d_{\text {corr }}}} Z_{01}+\sqrt{\frac{x}{d_{\text {corr }}}} Z_{11}\right)
\end{gathered}
$$

3. Uniform random variable can be obtained as a normalized angle of the interpolated complex variable $Z(x, y)$

$$
U(x, y)=\left|\frac{\angle \mathrm{Z}(\mathrm{x}, \mathrm{y})}{\pi}\right| .
$$

4. The LOS probability can be predicted by comparing a given $p_{\text {LOS }}$ probability with uniform distribution $U(x, y)$.

The results of LOS probability distribution for decorrelation distances of $10 \mathrm{~m}$ and $40 \mathrm{~m}$ are shown in Fig. 1. It shows LOS probability decaying with distance from central point as well as maintaining the granularity of spatial correlation pattern according to the given decorrelation distance.

\section{DUAL ENVIRONMENT LOS MODEL}

To better represent real propagation environments which usually contains inhomogeneous building blocks of varying height, we propose a combined dual environment model which approximates LOS probability over distance using two probability functions, one for each environment. In this section, details are given for such model implementation.

\section{A. Reconciliation with Grid Models}

The main goal of LOS/NLOS probability application in extending SEAMCAT functionality - is to find the best way to exploit Madrid/Manhattan grid for statistical simulation of LOS/NLOS states. Purely deterministic way would be deriving LOS visibility from building geometry, however it would not agree with statistical approach. Therefore, largescale statistical distribution parameters should be extracted from Madrid/Manhattan grid for use in generation of random LOS state distribution with a given probability density $p_{\mathrm{LOS}}(d)$. Along these ideas known methods of obtaining empirical parameters of $p_{\mathrm{LOS}}(d)$ distribution exist in the published works. The LOS probability parameters can be extracted from measurements [8] or obtained via simulations of radio path blockage either by ray tracing [9] or recently becoming popular point cloud data method [10]. When exploiting LOS probability and distance-dependent path loss models, Madrid/Manhattan grid structure parameters sometimes denoted as $d_{1}-d_{2}$ could be used as additional input parameters. E.g. [2] use street distance parameters depending on the direction of street derived from Manhattan grid geometry, or in [11] the same parameters are used for transition function between LOS and NLOS regions.
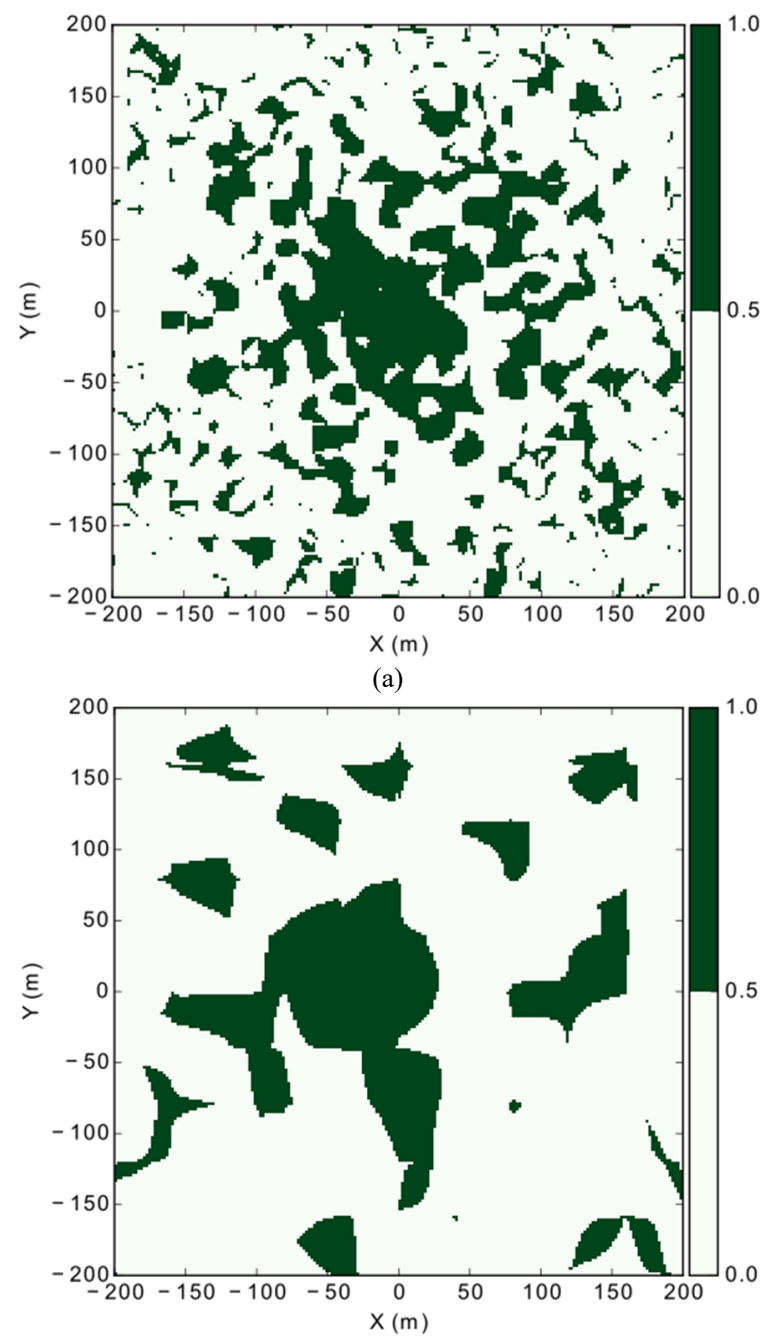

(b)

Fig. 1. Generated LOS probability distribution for decorrelation distance equal to: a) $10 \mathrm{~m}$ and; b) $40 \mathrm{~m}$

\section{B. Combining Different LOS Regions Using Transition Functions}

Transition functions between LOS and NLOS regions can be used as approximations to Fresnel diffraction integrals proposed in COST 259 directional channel model [12]. Following COST 259 channel model proposal, the following approximation for calculating soft LOS state probability is used

$$
p_{\text {LOS soft }}(x)=\frac{1}{2}-\frac{1}{\pi} \arctan \left[\sqrt{\frac{|x|}{\lambda}} \operatorname{sgn}(x)\right],
$$

where $x$ is a distance to LOS/NLOS boundary and $\lambda$ is the 
wavelength of central carrier.

For numerical implementation of soft LOS state probability, the shortest distance from LOS/NLOS boundaries to each location point has to be estimated. The LOS/NLOS boundaries are irregular, but can be approximated by polygons and the distance estimation reduces to calculating the distance between polygon and each location point. The distance of points inside the polygons are considered with negative sign. The results of distance estimation for a specific LOS/NLOS state distribution is given in top image of Fig. 3. The corresponding soft LOS state probability estimated using (6) is shown in bottom image of Fig. 3 .

\section{Combining Different Propagation Environments}

Marching squares algorithm has been applied to find the boundaries between obstructed and unobstructed propagation environments. It is a special case of the marching cubes algorithm adapted in two-dimensional plane [13].

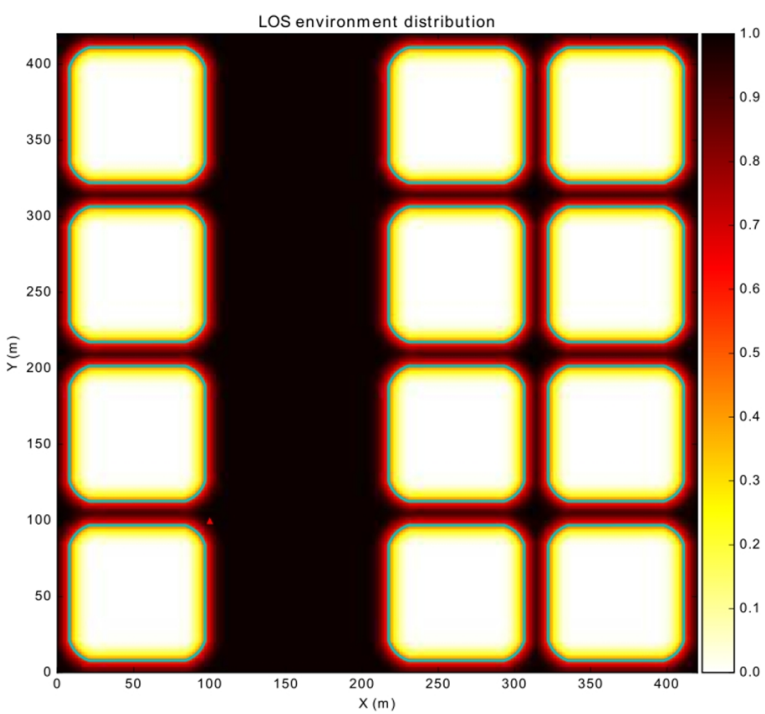

(a)

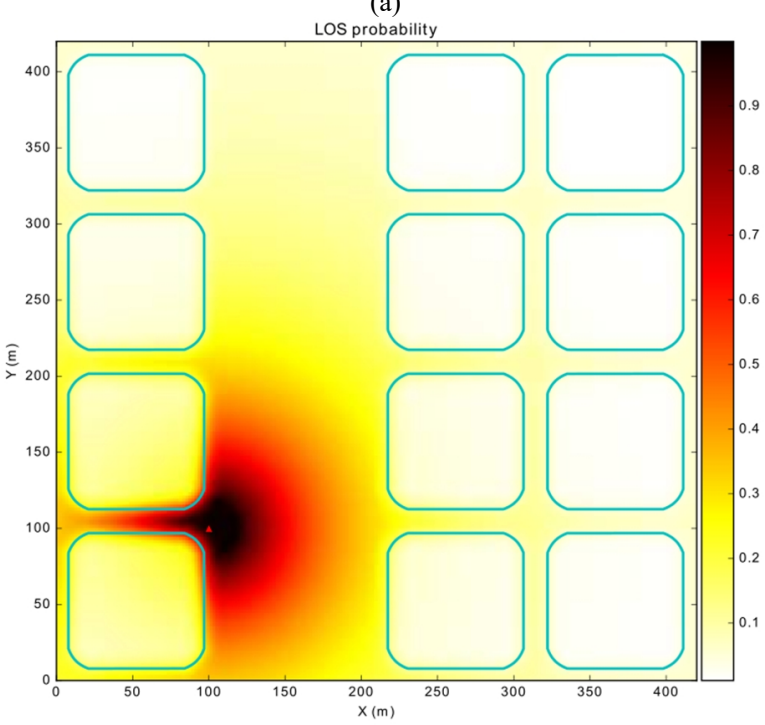

(b)

Fig. 2. Probabilistic distribution of LOS environment (a) and continuous LOS probability (b) for building Manhattan grid distribution.

Considering continuous distribution of blockage centers, the spatial distribution of centers with respect to mean blockage height $\mu_{h}$ and standard deviation $\sigma_{h}$ can be expressed by complementary cumulative distribution function for normally distributed heights as [14]

$$
f(x)=1-\frac{1}{2}\left[1-\operatorname{erf}\left(\frac{h(x)-\mu_{h}}{\sqrt{2} \sigma_{h}}\right)\right] .
$$

Then the composite LOS probability having two separate regions with different LOS probabilities $p^{(1)} \operatorname{LOS}(d)$ and $p^{(2)} \operatorname{Los}(d)$ can be estimated as

$$
p_{\mathrm{LOS}}(d)=p_{\mathrm{LOS}}^{(1)}(d) f(x)+p_{\mathrm{LOS}}^{(2)}(d)(1-f(x)),
$$

where $f(x)$ in this case indicates intensity of scattering centers in zone 1 with LOS probability $p^{(1)} \operatorname{Los}(d)$ at distance $d$ from the base station, while $x$ denotes the shortest distance to the boundary of zone 1 .

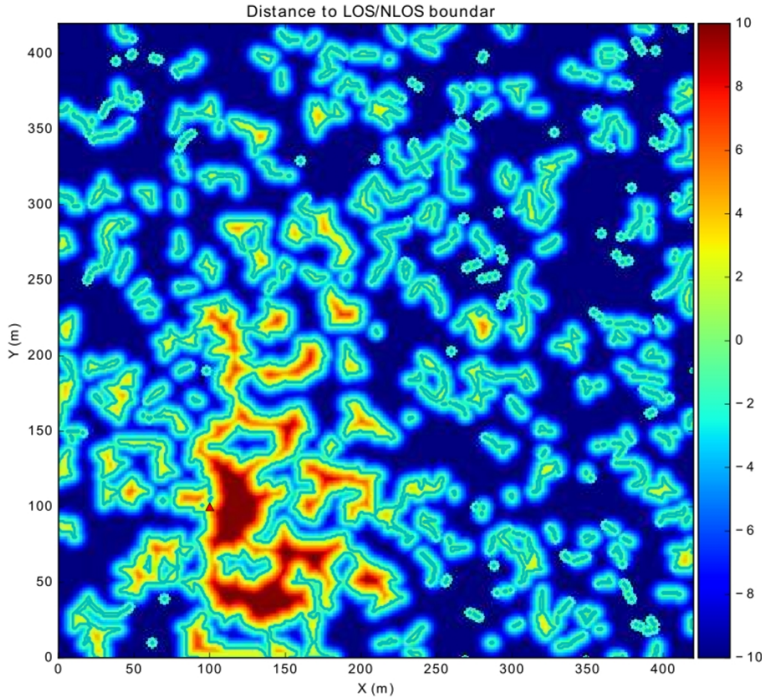

(a)

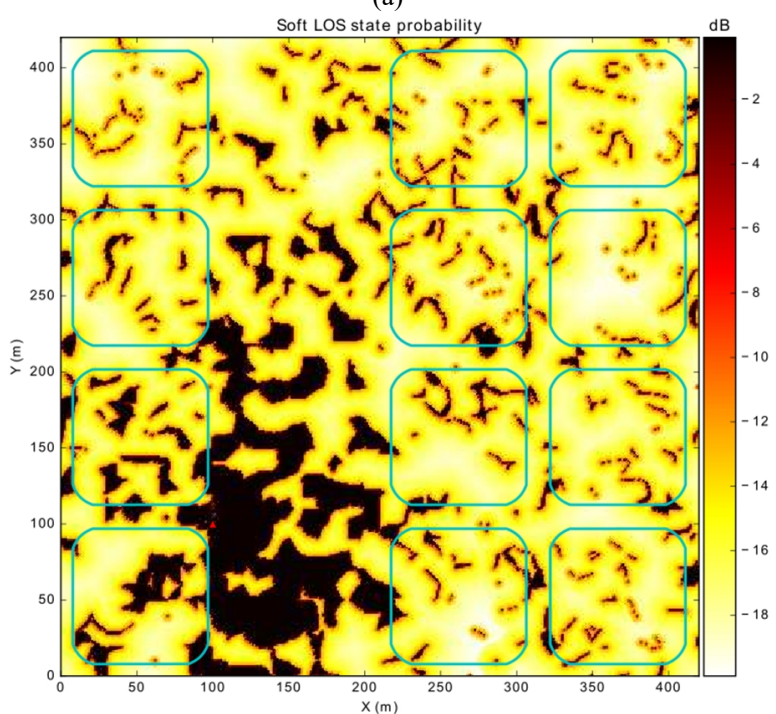

(b)

Fig. 3. Distance to LOS/NLOS boundaries (a) for a specific snapshot of LOS probability distributions and corresponding soft LOS probability (b) estimated using the distances to LOS/NLOS boundaries.

Building heights grid can be generated using non-uniform distributions. One example is normally distributed building heights within a city block, another is a city block with highest buildings at the center of a block with random height gradient direction from outskirts towards the center. 


\section{LOS Probability Approximation Based on Digital Building Model}

The correctness of proposed probability model can be verified against real city distributions. We will use extracts of Manhattan city building data referenced also in [15] in order to validate the proposed statistical visibility probability model against deterministic geometry-based visibility results. Our method of validation differs from that proposed in [15] in that we use raster-based operations instead of vector-based analysis. Converting vector data to matrix (raster) type format discards geometrical elements less than the matrix pixel size, but by adjusting the resolution of the matrix, geometric features in matrix format can be reproduced with desired accuracy. Then the matrix based analysis is more convenient in comparison to simplified version of vector data processing [15] which relies on certain kind of vector data processing approximations.

Visibility regions for matrix-based building data are generated according to geometrical considerations - by assessing line-of-sight point-to-point transmission lines between given base station antenna height and the highest obstacle. Thus LOS conditions are assumed for propagation paths with positive clearance distance.

The LOS distribution highly depends on the location of base station antenna and open line-of-sight area around the site. We identify three typical cases:

1. with open circular region around base station,

2. with half space open LOS when base station antenna is mounted on the rooftop at the edge of building block, and 3. without LOS region close to base station and LOS/NLOS state distribution is homogeneous throughout the whole base station coverage area.

These three typical LOS conditions for different Manhattan city locations are illustrated in the Fig. 4 and Fig. 5, respectively. Each set of images includes line-ofsight visibility around base station, building heights with buffer zones around them and the continuous distribution function (7) separating two LOS environments on each side of the buffer line.

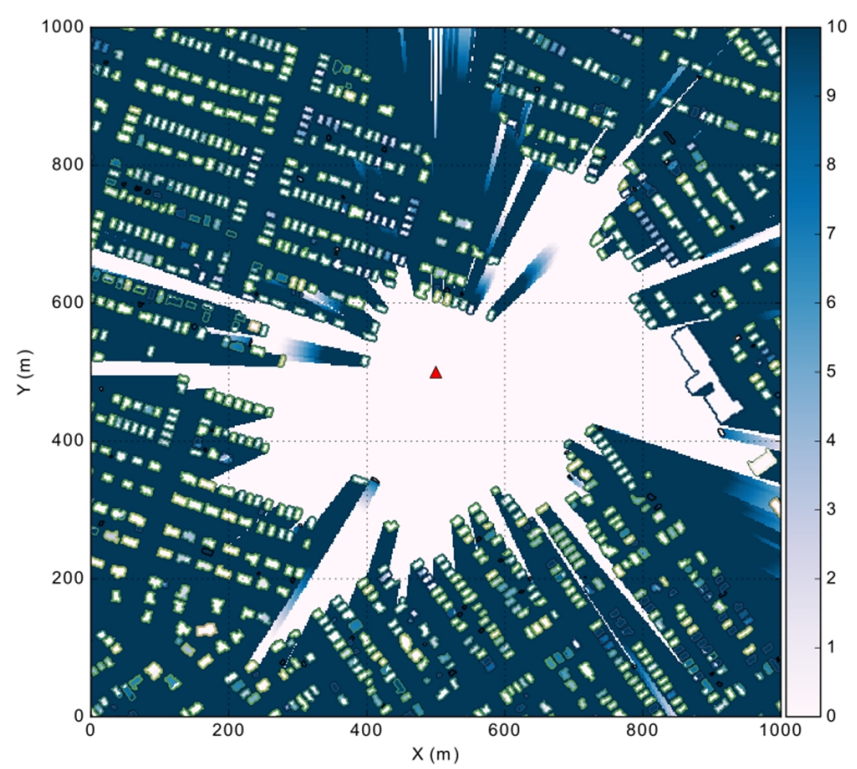

(a)

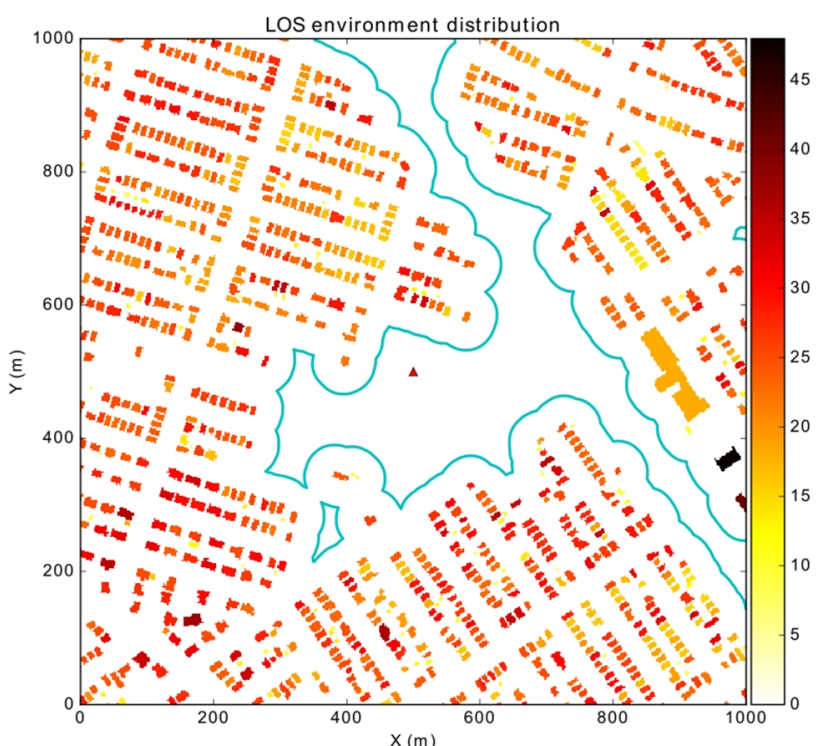

(b)

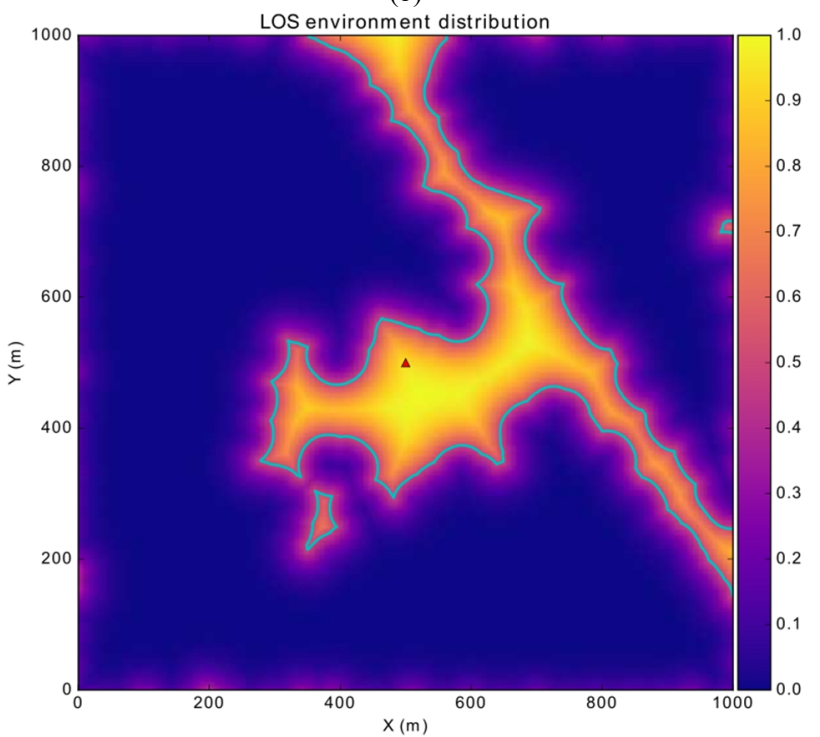

(c)

Fig. 4. Dual LOS environment separation for Manhattan city digital building model. The three images represent area with open circular region mentioned in the text as case 1. a) Represents line-of-sight visibility for each case, the numerical values express height in meters below visibility line, visible areas shown in white. b) Represents building heights in meters and boundary zones around buildings lower than $3 \mathrm{~m}$ with buffer zone of $40 \mathrm{~m}$. c) shows us the continuous distribution function (7) for the two LOS environments.

Least square parameter optimization has been applied to urban 3GPP LOS probability model (1) and to the combined dual environment LOS model represented by (8) substituting urban (1) and (2) 3GPP models for dual environment LOS probabilities $p^{(1)}$ LOS and $p^{(2)}$ LOS, respectively. The results of () $\left\{d_{1}, d_{2}, d_{3}, d_{4}\right\}$ parameter optimization is shown in Table I. The approximation accuracy of dual environment model over 3GPP LOS probability model is significantly better for the cases of open and semi-open visibility zone around base station. When there is no open visibility region near base station location, approximation result of both single and dual environments models are almost the same, with RMSE on the level of about 0.09 . In the case of open LOS visibility zone, dual environment model breaks up the azimuth angle symmetry and environment map becomes a part of LOS probability model as an external parameter. 


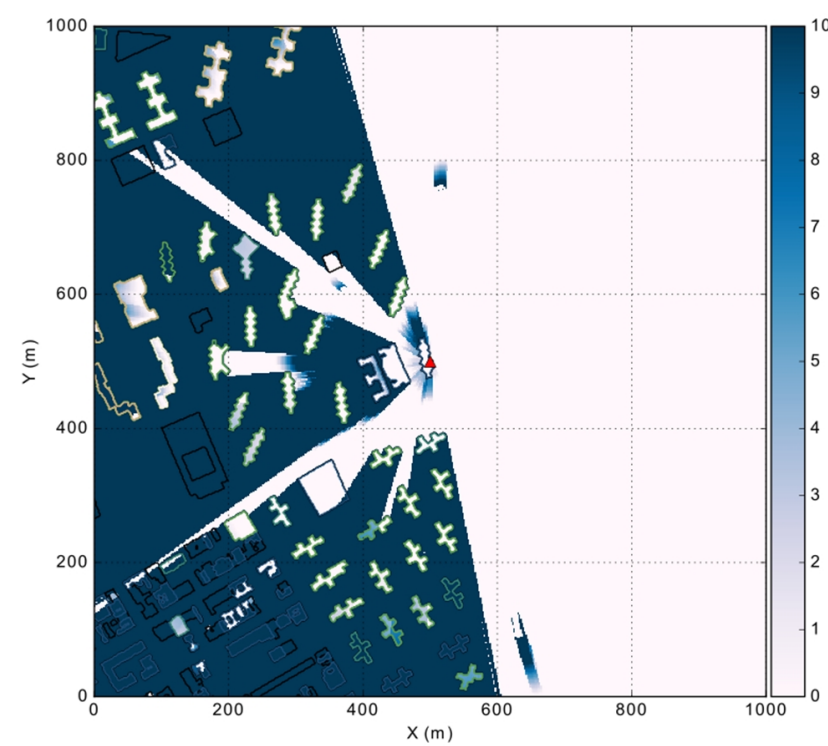

(a)

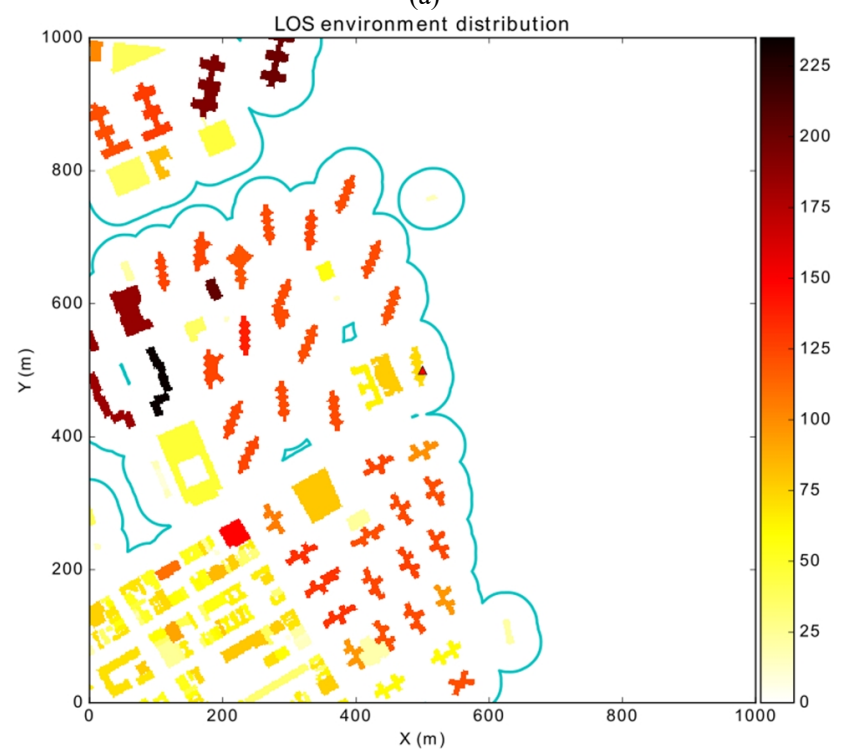

(b)

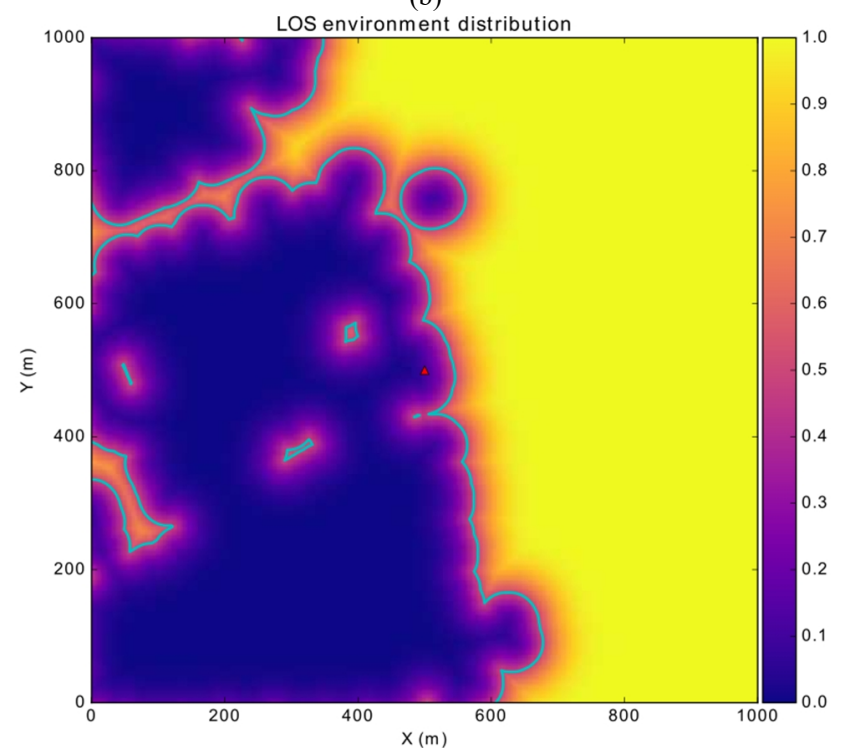

(c)

Fig. 5. Dual LOS environment separation for Manhattan city digital building model. The three images represent half space open LOS area or case 2. a) represents line-of-sight visibility for each case, the numerical values express height in meters below visibility line, visible areas shown in white. b) represents building heights in meters and boundary zones around buildings lower than $3 \mathrm{~m}$ with buffer zone of $40 \mathrm{~m}$. c) shows us the continuous distribution function (7) for the two LOS environments.
To make model more general we used trigonometric series approximation to the boundary $r_{\mathrm{bnd}}(\theta)$ of the nearest LOS zone as

$$
r_{\text {bnd }}(\Theta)=\sum_{n=0}^{N} a_{n} \sin (n \Theta)+b_{n} \cos (n \Theta),
$$

where $\theta$ is a polar azimuth angle around base station location. As shown in Table I, the approximation error for bounded LOS zone reaches RMSE 0.034 and becomes better than approximation with buffered environment zone. This is because zone boundary filters out farther open environment segments which in reality do not contribute to LOS visibility.

TABLE I. OPTIMIZATION RESULTS FOR LOS PROBABILITIES USING 3 GPP AND DUAL ENVIRONMENT MODELS.

\begin{tabular}{|c|c|c|c|c|c|c|}
\hline Scenario & Case & $d_{1}$ & $d_{2}$ & $d_{3}$ & $d_{4}$ & RMSE \\
\hline \multirow{4}{*}{1} & 3GPP default & 18.00 & 63.00 & - & - & 0.295 \\
\hline & $\begin{array}{c}\text { 3GPP } \\
\text { optimized }\end{array}$ & 60.30 & 156.93 & - & - & 0.107 \\
\hline & $\begin{array}{c}\text { Dual } \\
\text { environment }\end{array}$ & 282.96 & 22.12 & 1.45 & 136.77 & 0.045 \\
\hline & $\begin{array}{c}\text { Dual bounded } \\
\text { env }\end{array}$ & 1.00 & 53.37 & 6.14 & 101.39 & 0.034 \\
\hline \multirow{3}{*}{2} & 3GPP default & 18.00 & 63.00 & - & - & 0.525 \\
\hline & $\begin{array}{c}\text { 3GPP } \\
\text { optimized }\end{array}$ & 115.76 & 368.52 & - & - & 0.164 \\
\hline & $\begin{array}{c}\text { Dual } \\
\text { environment }\end{array}$ & 153.40 & 717.85 & 0.66 & 246.87 & 0.043 \\
\hline \multirow{3}{*}{3} & 3GPP default & 18.00 & 63.00 & - & - & 0.102 \\
\hline & $\begin{array}{c}\text { 3GPP } \\
\text { optimized }\end{array}$ & 1.00 & 83.59 & - & - & 0.089 \\
\hline & $\begin{array}{c}\text { Dual } \\
\text { environment }\end{array}$ & 1.00 & 7.16 & 1.10 & 80.48 & 0.086 \\
\hline
\end{tabular}

\section{CONCLUSIONS}

A statistical line-of-sight visibility state model is proposed involving dual environments characterized by different obstacle heights. The method allows to combine two probability functions similar to those used by ITU and 3GPP models. This improves approximation accuracy with respect to deterministic LOS estimation using digital building model. From the comparison of $3 \mathrm{GPP}$ and dual environment model approximations applied to Manhattan city building data it is evident that dual environment model is more accurate for areas enclosing LOS visibility areas around base station. Advantages of dual environment LOS model is especially obvious for base station locations at the boundary of LOS and NLOS regions when antenna is placed on the rooftop of the buildings at the edge of city blocks. The only disadvantage of the proposed LOS model is increased complexity when at least two LOS probabilities are combined thus increasing the number of $p_{\mathrm{LOS}}(d)$ coefficients. Dual environment approximation could be applied to other types of known LOS probability functions and more tests against diverse urban and suburban environments would enable reaching more quantitative results.

\section{REFERENCES}

[1] SEAMCAT - Spectrum Engineering Advanced Monte Carlo Analysis Tool. [Online]. Available: http://www.seamcat.org.

[2] ITU, "Guidelines for evaluation of radio interface technologies for IMT-Advanced", Report ITU, vol. M.2135, no. 1, p. 72, 2009.

[3] 3GPP TR 36.814, "Evolved Universal Terrestrial Radio Access (EUTRA); Further advancements for E-UTRA physical layer aspects", 
3GPP, Tech. Rep. Version 9.2.0, 2017. [Online]. Available: http://www.3gpp.org/dynareport/36814.htm.

[4] B. Mondal, T. A. Thomas, E. Visotsky, F. W. Vook, A. Ghosh, Y. H. Nam, Y. Li, J. Zhang, M. Zhang, Q. Luo, Y. Kakishima, K. Kitao, "3D channel model in 3GPP", IEEE Communications Magazine, vol. 53, no. 3, pp. 16-23, 2015. DOI: 10.1109/MCOM.2015.7060514.

[5] M. Samimi, T. Rappaport, G. Maccartney, "Probabilistic omnidirectional path loss models for millimeter-wave outdoor communications", IEEE Wireless Communications Letters, vol. 4, no. 4, pp. 357-360, 2015. DOI: 10.1109/LWC.2015.2417559.

[6] M. Gudmundson, "Correlation model for shadow fading in mobile radio systems", Electronics Letters, vol. 27, no. 23, pp. 2145-2146, 1991. DOI: $10.1023 / \mathrm{A}: 1012771509178$.

[7] Aalto University, AT\&T, BUPT, CMCC, Ericsson, Huawei, Intel, KT Corporation, Nokia, NTT DOCOMO, New York University, Qualcomm, Samsung, University of Bristol, University of Southern California, "5G Channel Model for bands up to $100 \mathrm{GHz}$," Tech. Rep. Version 2.3. [Online]. Available: http://www.5gworkshops.com /5GCM.html.

[8] J. Poutanen, K. Haneda, J. Salmi, V. M. Kolmonen, P. Vainikainen, "Modeling the evolution of number of clusters in indoor environments", in Proc. Fourth European Conf. Antennas and Propagation, 2010, pp. 1-5.

[9] Z. Zhang, J. Ryu, S. Subramanian, A. Sampath, "Coverage and channel characteristics of millimeter wave band using ray tracing", in IEEE Int. Conf. Communications (ICC 2015), 2015, pp. 1380-1385. DOI: 10.1109/ICC.2015.7248516.
[10] J. Jarvelainen, S. L. H. Nguyen, K. Haneda, R. Naderpour, U. T. Virk, "Evaluation of millimeter-wave line-of-sight probability with point cloud data", IEEE Wireless Communications Letters, vol. 5, no. 3, pp. 228-231, 2016. DOI: 10.1109/LWC.2016.2521656.

[11] A. Molisch, H. Asplund, R. Heddergott, M. Steinbauer, T. Zwick, "The COST259 directional channel model-Part I: Overview and methodology", IEEE Trans. Wireless Communications, vol. 5, no. 12, pp. 3421-3433, 2006. DOI: 10.1109/TWC.2006.256966.

[12] H. Asplund, A. F. Molisch, M. Steinbauer, N. B. Mehta, "Clustering of scatterers in mobile radio channels-evaluation and modeling in the COST259 directional channel model", in IEEE Int. Conf. Communications (ICC 2002), 2002, pp. 901-905. DOI 10.1109/ICC.2002.996986.

[13] W. E. Lorensen, H. E. Cline, "Marching cubes: a high resolution 3D surface construction algorithm", in Proc. 14th Annual Conf. Computer Graphics and Interactive Techniques, (SIGGRAPH 1987). New York, NY, USA, 1987, pp. 163-169. DOI: $10.1145 / 37401.37422$

[14] M. Gapeyenko, A. Samuylov, M. Gerasimenko, D. Moltchanov, S. Singh, E. Aryafar, S. p. Yeh, N. Himayat, S. Andreev, Y. Koucheryavy, "Analysis of human-body blockage in urban millimeter-wave cellular communications", in IEEE Int. Conf Communications (ICC 2016), 2016, pp. 1-7. DOI: 10.1109/ICC.2016.7511572.

[15] J. G. Andrews, T. Bai, M. N. Kulkarni, A. Alkhateeb, A. K. Gupta, R. W. Heath, "Modeling and analyzing millimeter wave cellular systems", IEEE Trans. Communications, vol. 65, no. 1, pp. 403-430 2017. DOI: $10.1109 /$ TCOMM.2016.2618794 